Dessalles, J-L. (1999). Coalition factor in the evolution of non-kin altruism. Advances in Complex Systems, 2 (2), $143-172$. Available at: http://www.dessalles.fr/papers/Dessalles_99091402.pdf

\title{
Coalition factor in the evolution of non-kin altruism
}

\author{
Jean-Louis Dessalles \\ Ecole Nationale des Télécommunications \\ e-mail: dessalles@enst.fr
}

\begin{abstract}
Animal behavior is often altruistic. In the frame of the theory of natural selection, altruism can only exist under specific conditions like kin selection or reciprocal cooperation. We show that reciprocal cooperation, which is generally invoked to explain non-kin altruism, requires very restrictive conditions to be stable. Some of these conditions are not met in many cases of altruism observed in nature. In search of another explanation of non-kin altruism, we consider Zahavis's theory of prestige. We extend it to propose a 'political' model of altruism. We give evidence showing that non-kin altruism can evolve in the context of inter-subgroup competition. Under such circumstances, altruistic behavior can be used by individuals to advertise their quality as efficient coalition members. In this model, only abilities which positively correlate with the subgroup success can evolve into altruistic behaviors.

KEYWORDS: altruism, evolution, cooperation, status, coalitions.
\end{abstract}

\section{Introduction: altruism and natural selection}

The existence of altruistic behaviors in the animal kingdom seems at first sight to challenge Darwin's theory of natural selection. An individual's behavior should be consistent with the spreading of its genes. Altruistic behavior, however, results inothers being offered a better chance to spread their own genes at the expense of the altruistic individual. The study of insect societies led to a first explanation of this contradiction. The concept of kin selection explains how altruistic behavior can lead to a better spreading of genes if it is directed toward genetically related individuals who are likely to have copies of the altruistic individual's genes (Hamilton 1964).

Kin selection will not be our concern here. We will focus on situations in which non-related individuals behave altruistically to each other. Non-kin altruism is to be observed in animal and human behavior. Human beings are indeed able to 
bring significant help to each other well beyond the family context. If we accept that such abilities have a biological basis, then they require a Darwinian account that makes them compatible with the principles of natural selection.

There has been much debate on how to know whether non-kin altruism could be explained in terms of group selection or of 'preservation' of species (Williams 1966, Wilson and Sober 1994). Such explanations must avoid an obvious flaw: within the group, both altruistic and non-altruistic individuals benefit from collective advantages induced by altruistic acts. Altruistic behavior, as soon as it involves a cost, should thus disappear in the process of within-group selection. As a consequence, genuine group selection of altruistic traits can only exist in very specific situations in which (1) individual success is strongly related to intergroup competition and (2) the variability of reproductive success within the group is relatively small (Jones 1996, Dessalles 1996). Unfortunately, non-kin altruism can be found in cases where these requirements are not met, especially in the case of human evolution (Palmer et al. 1996).

We are traditionally left with one single explanation of such non-kin altruistic behavior. It would be based on reciprocation (Trivers 1971). The basic idea is that since it is often profitable for two individuals to cooperate, what appears as altruism is in fact one half of a cooperative transaction. If A gives $G_{1}$ to $\mathrm{B}$ and bears the $\operatorname{cost} C_{1}$, the behavior seems altruistic, but if B spends $C_{2}$ to return $G_{2}$ to A, both participants benefit from the transaction as soon as $G_{2}-C_{1}>0$ and $G_{1}-$ $C_{2}>0$. The main problem with cooperative behavior, however, is that it is sensitive to defection. Since A and B's acts are most often not simultaneous, B may be tempted not to reciprocate in order to gain $G_{l}$ in full, which causes A not to get $G_{2}$. Cooperative behavior can only evolve together with a cheating detection device that allows cooperative individuals to check the partner's willingness to play the game according to the rules.

Amotz Zahavi recently proposed a new explanation of some form of altruistic behavior (Zahavi 1995; Zahavi and Zahavi 1997). His field observations, while supporting reciprocal altruism at first sight, reveal facts that are incompatible with it. For instance babblers, a species of small birds, show strange behaviors: they literally compete to be altruistic. According to Zahavi, altruistic individuals are rewarded by gaining 'prestige'.

In this paper, we compare these two alternative explanations of non-kin altruism: reciprocal cooperation and Zahavi's theory of prestige. We designed models of both phenomena so that they could be easily compared on a theoretical basis and through computer simulations. In Section 2, we show that reciprocal altruism can be evolutionary stable and may create a selection pressure towards cooperation, but that very specific conditions are required for this to happen. Since such requirements are very restrictive, we consider Zahavi's model as an alternative in Section 3. We show, by means of computer simulations, that a straightforward implementation of Zahavi's theory of prestige cannot account for the evolution of altruism among non-related individuals. In Section 4, we show how the introduction of a coalition factor in Zahavi's model leads to a sound scenario of the evolution of non-kin altruism. We will develop what we call a 'political' model of altruism, in which the role of altruistic behavior is to advertise an ability which is useful in inter-coalition competition. 


\section{Altruism as cooperation}

Biological altruism is a complex issue. Living beings are involved in ecological contexts which include other individuals of the same species and of different species. Many random events of various importance perturb inter-individual interactions and modify their consequences. Altruistic behaviors themselves are never simple: they are performed under specific conditions and may take various forms when analyzed in sufficient detail. However, any sound understanding of the genetic mechanisms that allow altruism to exist in nature requires that we make drastic simplifications. The computational models described in this paper implement versions of altruism which may appear as simplistic from a biological perspective. Nevertheless, they prove useful to isolate relevant determinants of altruism. Moreover, they put constraints on what biological accounts can be: they cannot include mechanisms that are proven unsound by computer simulations.

\subsection{THE EVOLUTION OF COOPERATION}

Determining the exact conditions that make cooperation possible is a crucial issue, both in social sciences like economics and in evolutionary biology. It has been the object of an extended literature. The problem for an agent of choosing between being cooperative or uncooperative crucially depends on others' strategies. The stability of strategies in the long term is thus problematic (Axelrod and Hamilton 1981; Axelrod 1984). Most models designed for the study of cooperation strategies are based on an iterated version of the prisoner's dilemma (IPD). Individuals are randomly or systematically chosen to play a cooperative two-part game, in which both partners decide to be either cooperative or uncooperative. Individuals are supposed to be cognitive agents, endowed with enough memory to keep track of their previous encounters with specific individuals. This allows complex strategies to develop in a multi-agent context. However, simple strategies like the classical tit-for-tat (TFT) were shown to be quite successful in the long run in the absence of noise (Axelrod 1984).

TFT is not systematically cooperative. Its first move with an unknown partner is cooperative, but then TFT adopts the same attitude as its opponent's last move. TFT can thus be very unfriendly toward uncooperative opponents. When noise is present, i.e., when moves can be misunderstood, several TFTs can be uncooperative to each other. In such an unpredictable environment, without further restriction, cooperation does not emerge. Many attempts have been made to find specific contexts in which cooperation appears and remains stable. One of them is to put agents in a space in such a way that neighbors are more likely to interact. In such situations, defecting strategies may prove to be less successful than cooperative ones (Axelrod 1984:158; Ferriere and Michod 1996; Nakamaru et al. 1997).

Classical implementations of IPD have three characteristics: (i) agents merely accumulate points, until the tournament is over; (ii) pairs of players in each iteration are randomly chosen; (iii) the behavior of each agent is adapted to its opponent's past behavior. However, this standard approach to the study of cooperation is not universal. Some models are more biologically inspired than (i) and make use of genetic algorithms: strategies reproduce or die according to their success. This allows us to use simulation to understand biological cooperative 
behaviors (Key and Aiello, in press). Some studies depart from (ii) by distinguishing an 'assortative' phase, during which partners choose each other, from the cooperative game itself (Dugatkin 1997, Cooper and Wallace 1998). In most models, the probability that partners separate does not depend on their cooperation. In (Peck 1993), however, individuals may break unprofitable partnerships. Property (iii) seems to be essential. However, it presupposes that agents are sophisticated enough to keep track of past encounters with specific individuals and to make non-trivial computations before making their decision, using concepts like reward, deception and retaliation. Some studies of IPD (e.g., Ferriere and Michod 1996; Peck 1993) consider only simple and systematic strategies like 'always defect' and TFT. The problem is then to find conditions that allow TFT to survive or to invade the population. Some studies (e.g., Frean 1996) consider simple strategies, but these strategies depend on gradual parameters.

Our purpose in this paper is not to study cooperation per se, but to investigate the conditions under which it can emerge and remain evolutionary stable. Our own model thus departs from properties (i)-(iii): we adopt a genetic algorithm implementation, partners choose each other up to some point, and only basic cooperation is available to them. We opted for radical versions of these three options. Our implementation of genetic algorithms is biologically inspired: the population is structured in groups, mating and interactions take place within the group, individuals are generated through conventional crossover between the binary genomes of their parents, they are characterized by their age, their vitality, and they may undergo random accidents. Partnership is flexible: agents may leave their usual partner if they meet a more responsive individual. Lastly, cooperative strategies are simpler than in most implementations. All agents have basically the same behavior: they offer vitality points to the partner, at their own expense, and the partner responds by giving a fraction of these points in return, at its own expense too. Thus the initiator controls the stake, while the respondent controls the cooperation. These behaviors are stereotyped, but gradual. Bids and responses are controlled by genetic coefficients which may take various values (typically 256). Our aim is not only to check whether cooperation is evolutionary stable, it is also to determine which cooperation level does emerge. The gradual set of strategies serves the latter purpose.

Since individual strategies are genetically determined in our simulation, agents cannot adopt even simple strategies like TFT, since they cannot change their investment when the partner is unresponsive. However, as soon as they are involved in a more profitable interaction, they may break partnership and designate the new individual as their preferred partner. In classical implementations, strategies are flexible, while partnership is either random or fixed. Our model is characterized by fixed strategies and flexible partnership. The main reason for making these choices is simplicity: complex cooperative strategies are unlikely to evolve from scratch. There must be some simple precursors which play a scaffolding role. These precursors must be proven evolutionary stable for more complex strategies to be imaginable.

\subsection{A GENETIC MODEL OF COOPERATION}

The characteristics of our model, which are detailed below, require minimal cognitive abilities: 
- Interactions: Each agent initiates several interactions each 'year', with individuals of the same group. The partner returns a fraction (possibly bigger than 1) of the amount received. All agents in turn play the first part with some partners. Cooperation, i.e., the amount returned by the second player, is proportional to the initial gift of the first player. Costs supported by both players are also gradual, proportional to what they give.

- Individuals: Agents' strategies are genetically fixed. A fixed number of bits (from 2 to 32) is devoted to the definition of two characteristic parameters: (1) a coefficient $g_{l}$ affecting the amount of 'life points' given by the agent when playing first; (2) a coefficient $g_{2}$ determining the cooperative attitude when the agent is in a situation of playing the second move. When an agent A gives $G g_{1 A} n$ to B, it receives $R g_{2 B} g_{1 A} n$ in return. $G$ and $R$ are constants, and $n$ is a noise modulation. Since $g_{1}$ and $g_{2}$ are coded with several bits (in all simulations presented here, we used the conventional binary code for integers), both strategies are gradual, i.e., there is a spectrum of different possible strategies in the population. Agents reproduce as usual in genetic algorithms (Goldberg 1989; Dessalles 1996), so that an offspring's strategy is a hybrid resulting from a crossover between the genomes defining its parents' strategies.

- Groups: The population is structured in semi-permeable groups. Games are played within groups. Reproduction also takes place between members of the same group. Each 'year', a fixed proportion of the population migrates, moving randomly to other groups. Groups have a maximal size, beyond which they split. This structuring in groups allows us to study the evolution of local cooperation.

- Partnership: Individuals have limited bookkeeping capabilities: they remember a characteristic of the most cooperative agent previously encountered during the 'year'. This characteristic is a four digit label, randomly assigned to individuals at birth (there are thus potentially $10^{4}$ different labels). For each cooperative game, candidates for playing the partner role are randomly chosen within the group until the memorized characteristic is recognized or until the procedure stops. If the group is small enough, there is thus a good probability that the preferred partner will be selected. The selection procedure is however quite noisy: with some probability, it may be prematurely stopped, thus offering an unexpected partner.

This model implements a gradual version of cooperation. The question is now whether such a system evolves toward cooperation, and what level this cooperation may reach. One can determine actual profits made by agents when playing the first or the second move (here $g_{1}$ is relative to the first individual and $g_{2}$ to the second one):

$$
\begin{aligned}
\text { Initiator's profit: } & g_{1} n\left(R g_{2}-C_{1}\right) \\
\text { Respondent's profit: } & g_{1} n\left(G-C_{2} g_{2}\right)
\end{aligned}
$$

with 
$G$ : coefficient for the computation of the initial gift (in 'life points') (constant)

$R$ : coefficient for the computation of the reward (constant)

$g_{I}$ : coefficient, between 0 and 1 , affecting the initial gift (genetically determined)

$g_{2}$ : coefficient, between 0 and 1 , affecting the reward (genetically determined)

$C_{1}$ : coefficient for the computation of the initiator's cost (constant)

$C_{2}$ : coefficient for the computation of the respondent's cost (constant)

$n$ : noise modulation (mean 1 ).

Average profits are easy to compute in an unstructured population, where individuals have a small probability of finding their 'preferred' partner. There is no correlation between $g_{l}$ and $g_{2}$ in the preceding expressions, since they concern different individuals. Expected profits are:

$$
\begin{aligned}
\text { Initiator's expected profit: } & N g_{1}\left(R g_{2 m}-C_{1}\right) \\
\text { Respondent's expected profit: } & N P g_{1 m}\left(G-C_{2} g_{2}\right) s
\end{aligned}
$$

where $g_{I m}$ and $g_{2 m}$ stand for the average value of $g_{1}$ and $g_{2}$ in the population. Rigorously, the individual considered is not included in the computation of the mean. When necessary, we will indicate this by inserting primes: $g_{1 m}^{\prime}$ and $g^{\prime}{ }_{2 m}$. $N$ is the number of interactions initiated by one individual each year. $s$ is the probability of an individual being chosen as partner, and $P$ is the group size. NPS is the expected number of interactions where a given individual plays the respondent's role. In a large group, $s=1 / P$, since the partner choice is nearly random. We see that under such circumstances, $g_{2}$ will evolve to be as small as possible, since small $g_{2}$ make bigger profits. As a consequence, $g_{l}$ will tend to be small too, in order to minimize the first player's loss. Cooperation does not exist there.

Things are different when interactions take place within groups and when first players are offered the possibility of remembering their best partner. If we take this notion of preferred partner into account, the probability for a given agent of being selected as respondent in an interaction is no longer a constant $s$. Each initiator, through its encounters, realizes a limited sampling of the group, remembering the best value of $g_{2} n$. The probability for a given individual to be chosen as favorite partner depends on the rank of its own $g_{2}$ in the $g_{2} n$ distribution. Applying Markov's inequality, the probability that another individual characterized by $g_{2}^{\prime}$ is preferred can be majored: $\operatorname{Prob}\left(g_{2}^{\prime} n^{\prime}>g_{2} n\right)=$ $\operatorname{Prob}\left(g_{2}^{\prime} n^{\prime} / n>g_{2}\right) \leq g_{2 m}^{\prime} / g_{2}$. We see that when $g_{2} / g_{2 m}^{\prime}$ is high enough, the individual is likely to be selected. Under certain restrictive assumptions, the probability of being selected can be approximated as a function of $g_{2} / g^{\prime}{ }_{2 m}$. If $F\left(g_{2}\right)$ is the cumulative distribution of probability of $g_{2} n$, then $1-F\left(g_{2}\right)$ gives the probability of picking an individual which is more responsive than an individual endowed with $g_{2}$. The precise form of $F$ depends on experimental settings. Thanks to the presence of the noise $n$, however, we expect $F$ to be a spread out (increasing) function, typically an elongated sigmoid. A rough linear approximation of $F$ would give $F\left(g_{2}\right) \approx g_{2} / 2 g_{2 m}$, where the average $g_{2 m}$ is taken as the median: $F\left(g_{2 m}\right)=1 / 2$. For the sake of simplicity, we will consider accordingly that the probability of being respondent, which depends on $g_{2}$ through $F$, can be 
written as a function $s\left(g_{2} / g_{2 m}\right)$ of the relative value of $g_{2}$. As we will see, this approximation will not affect the qualitative reasoning that follows. The expected profits are now biased by the fact that initiators may discriminate their favorite partner:

$$
\text { Initiator's expected profit: } \quad N g_{1}\left(p R g_{2 M}+(1-p) R g_{2 m}-C_{1}\right)
$$

Respondent's expected profit:

$$
N P g_{1 m}\left(G-C_{2} g_{2}\right) s\left(g_{2} / g^{\prime}{ }_{2 m}\right)
$$

$p$ is the probability of an initiator finding its favorite partner. The procedure which consists in remembering a good partner and in trying to find it again in subsequent games retains individuals characterized by an average cooperation coefficient $g_{2 M}$. This allows initiators to make a positive profit, since $g_{2 M}$ may be sufficiently large to compensate for $\mathrm{C}_{l}$. The expected profit of respondents depends on the expected number of times they are selected as partner. This expected number is given by $N P s\left(g_{2} / g^{\prime}{ }_{2 m}\right)$.

Respondents are thus involved in a 'commercial' game. With a low $g_{2}$, they makea bigger profit, but fewer individuals hire their services, whereas with a high $g_{2}$, they have better chances of being selected, though their profit may reach a minimum. There is thus a situation of competition, and we expect $g_{2}$ to attain significant values. Things are not that simple, however. When groups are not too small, there is still a probability of being chosen by mere chance. When $g_{2 m}^{\prime}$ is large, the low $g_{2}$ strategy becomes preferable, since it is too costly to be above $g^{\prime}{ }_{2 m}$. As we will see, this can lead to oscillatory regimes, where $g_{1 m}$ and $g_{2 m}$ values fluctuate widely.

\subsection{EVOLUTIONARY STABILITY OF COOPERATION}

In order to determine the evolutionary stability of cooperation in our model, let us suppose that the system is able to reach a stable state in which $g_{1 m}=g_{1}{ }^{*}$ and $g_{2 m}=g_{2}{ }^{*}$. We must verify that these values maximize both (2.3) and (2.4). Expression (2.3) is linear in $g_{l}$, or almost linear if we consider that $g_{l}$ alone may have a slight influence on $g_{2 m}$. In any case, maximum values are obtained for $g_{l}{ }^{*}$ $=g_{\text {Imax }}$ or $g_{l}{ }^{*}=0$, depending on the sign of the expression. The derivative of (2.4) is:

$$
\begin{aligned}
N P g_{1 m}\left[-C_{2} s\left(g_{2} / g^{\prime}{ }_{2 m}\right)+\left(G-C_{2} g_{2}\right)\right. & \left.s^{\prime}\left(g_{2} / g^{\prime}{ }_{2 m}\right) / g^{\prime}{ }_{2 m}\right] \\
& +N P \partial g_{1 m} / \partial g_{2} \cdot\left(G-C_{2} g_{2}\right) s\left(g_{2} / g^{\prime}{ }_{2 m}\right)
\end{aligned}
$$

At equilibrium, we expect $g_{1 m}$ to stick to an extremal value of $g_{l}$, either 0 or $g_{\text {lmax }}$, and thus we expect $g_{2}$ to have little influence on it. We will thus neglect the third term in the preceding expression. A stable state requires the expression in square brackets to be zero. Its first term is negative; the second one must thus counterbalance it. In the model, $s^{\prime}\left(g_{2} / g^{\prime}{ }_{2 m}\right)$ is always positive (if $g_{2}$ increases, its bearer is more likely to be 'appreciated' as partner). Its precise value depends on the scenario chosen for the selection of partners. The point is that whatever this (positive) value is, there is always a value of $g^{\prime}{ }_{2 m}$ which is small enough to give the second term the required value. To put it differently, a small absolute 
change in $g_{2}$ is, relatively to $g^{\prime}{ }_{2 m}$ either negligible or noticeable, depending on the value of $g_{2 m}^{\prime}$. When $g_{2 m}^{\prime}$ is small enough, the global derivative can be positive: a small increase of $g_{2}$ significantly augments the probability of being selected while producing a low additional cost. We can thus conclude that $g_{2 m}$ never drops down to zero, and reaches an equilibrium $g_{2}{ }^{*}$. Notice that if $F$ is sigmoid, our linear approximation of $F\left(g_{2}\right)$ underestimates $F^{\prime}\left(g^{\prime}{ }_{2 m}\right)$, and thus when $g_{2}$ is close to $g^{\prime}{ }_{2 m}$, the derivative $s^{\prime}\left(g_{2} / g^{\prime}{ }_{2 m}\right) / g^{\prime}{ }_{2 m}$ underestimates the derivative of the probability of being selected, which depends on $g_{2}$ through $F$ (all derivatives are positive). Our approximation has no influence on the conclusion that $g_{2 m}$ stabilizes on a non-zero value $g_{2}^{*}$.

We can compute the value of $g_{2}{ }^{*}$. At equilibrium, $g_{2}=g_{2}{ }^{*}=g^{\prime}{ }_{2 m}$. The expression in square brackets is zero, which gives:

$$
g_{2}^{*}=\frac{G}{C_{2}\left(1+\frac{s(1)}{s^{\prime}(1)}\right)}
$$

At equilibrium, expression (2.3) becomes $\operatorname{Ng}_{1}{ }^{*}\left(\operatorname{Rg}_{2}{ }^{*}-C_{l}\right)$. This leads us to consider three cases.

Case 1 : Stable cooperation

When $g_{2}{ }^{*}$ is significantly higher than $C_{1} / R$, then $(2.3)$ is positive and $g_{1}{ }^{*}=g_{1 \max }$. The pair $\left(g_{\text {lmax }}, g_{2}{ }^{*}\right)$ defines an evolutionary stable strategy. Such a situation is made possible when:

$$
G R /\left(C_{1} C_{2}\right)>\left(1+s(1) / s^{\prime}(1)\right)
$$

This means that $G$ and $R$ have to be high and $C_{1}$ and $C_{2}$ low. Stable cooperation also requires that $s^{\prime}(1)$ have a significant value. This means that $g_{2}$ must have a definite influence on the probability of being selected as partner. The initiators' discriminative choice, which is possible when groups are not too large, is thus an essential part of the scenario.

\section{Case 2 : No cooperation}

When these conditions are not met so that $g_{2}{ }^{*}$ is significantly lower than $C_{1} / R, g_{1}{ }^{*}$ is zero and $g_{1 m}$ drops down to zero. The computation of $g_{2}{ }^{*}$ is no longer meaningful when $g_{I m}=0$. We see from (2.4) that $g_{2}$ becomes neutral. There is no possible cooperation in this case.

\section{Case 3 : Unstable cooperation}

There is a range of parameter values for which $g_{2}{ }^{*}$ is of the same order of magnitude as $C_{I} / R$. Interesting phenomena occur there. In experimental settings, there is a wide uncertainty about $s(1) / s^{\prime}(1)$. Both $s(1)$ and $s^{\prime}(1)$ indeed crucially depend on random events associated with the selection of partners. In groups of intermediate size, the hazards of individual encounters, when cumulated, deviate from the theoretical average. These fluctuations have a significant influence on local values of $g_{2}{ }^{*}$. Whenever the fluctuations of $g_{2}{ }^{*}$ change the sign of $g_{1}{ }^{*}\left(\operatorname{Rg}_{2}{ }^{*}-C_{1}\right), g_{1}{ }^{*}$ switches from $g_{\text {Imax }}$ to 0 . This has a decisive influence on $g_{I m}$ which oscillate to follow these switches. The system thus dramatically amplifies random fluctuations of $g_{2}{ }^{*}$. Also, when $g_{2}{ }^{*}$ is close to $C_{1} / R$, individual values of $g_{2}$ have a definite influence on $g_{1 m}$. 
This creates an additional cause for $g_{2}{ }^{*}$ to fluctuate, since the third term in the derivative of (2.4) can no longer be neglected. For all these reasons, uncertainty about $s$ and $s^{\prime}$ maintains wide oscillations of $g_{1 m}$ and $g_{2 m}$.

Computer simulations confirm this analysis. In Figure 1a, we are in a situation where $C_{1}$ is small while $R$ is significant. $g_{1 m}$ reaches maximal values, while $g_{2 m}$ stabilizes. In the situation of Figure $1 b$, the population consists of one single group of several hundreds of individuals. In such a large group, the probability of finding the preferred partner is small. The influence of $g_{2}$ on the probability of being selected (measured by $\left.s^{\prime}(1)\right)$ is consequently very small. The theoretical value of $g_{2}{ }^{*}$ is thus small too. We are in Case 2, and Figure $1 \mathrm{~b}$ confirms that there is no cooperation in such a situation: $g_{l m}$ does not reach significant values. Figure $1 \mathrm{c}$ illustrates the intermediate situation of Case 3. Groups are of average size as in (a), but the cost $C_{l}$ has an intermediary value. Both $g_{1 m}$ and $g_{2 m}$ widely oscillate in parallel. Figure 1d summarizes these results. The plot shows the average of $g_{1 \mathrm{~m}}$ over generations for several simulations with the same settings as in (a), but with different values for $C_{1}$. The three parts of the plot correspond to Cases 1,3 , and 2 respectively. In the middle area, the dispersion of values illustrates the fact that the time average of $g_{l m}$ is affected by the oscillatory regime shown in (c).

An important feature of these simulations is that the migration rate between groups is kept significant, typically over $10 \%$, which means that more than $10 \%$ of the individuals are randomly sent to another group at the end of each 'year'. The population is structured in groups in order to study spatial effects on the evolution of cooperation. Reproduction is supposed to take place in these groups too. If the migration rate between groups is low (typically $5 \%$ or less), what we observe is a kin selection effect, since individuals are likely to play cooperative games with their offspring, brothers or cousins. Genetic correlation in small groups may explain many cases of evolutionary stable cooperation observed in structured populations (Nakamaru et al. 1997; Ferriere and Michod 1996; Macy and Skvoretz 1998). Figure 2 shows the effect of the migration rate on cooperation. In Figure $2 \mathrm{a}, 5 \%$ of the population change group randomly each 'year'. An increase of this migration factor is sufficient to destabilize cooperation, as shown in Figure $2 \mathrm{~b}$. In order to avoid mixing the effects of kin-selection and cooperation, we performed all simulations with high migration rates. The results illustrated in Figure 1 are thus genuine cooperation effects.

\subsection{CONDITIONS OF COOPERATION}

Many studies of cooperation are concerned with the possibility of reaching equilibria characterized by a certain proportion of cooperative strategies. For instance, Peck (1993) shows that TFT may reach a definite proportion of the population when the probability of breaking partnership is allowed to vary in a certain range. However, such studies are not concerned with the dynamics of cooperation. Are theoretical equilibria reliable, or are they subject to important 


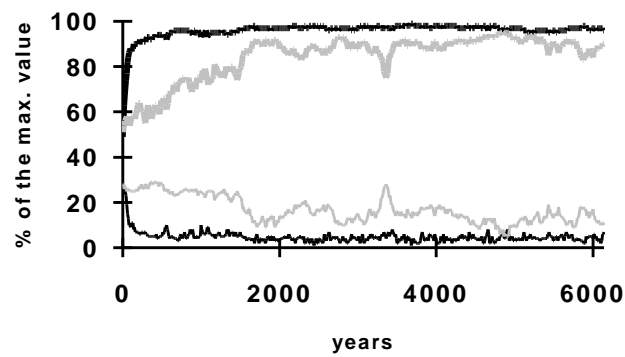

(a)

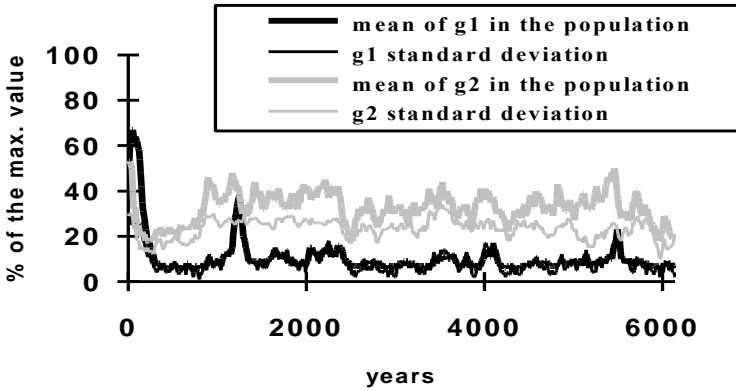

(b)

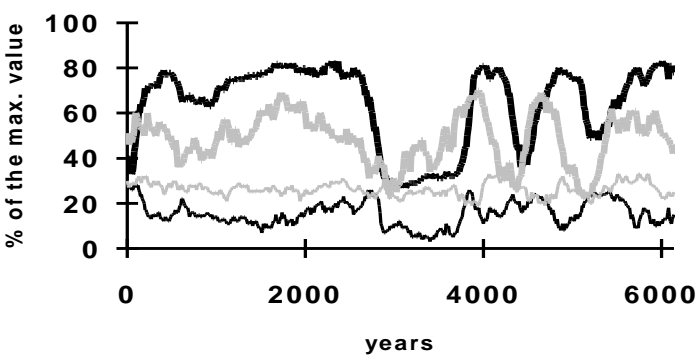

(c)

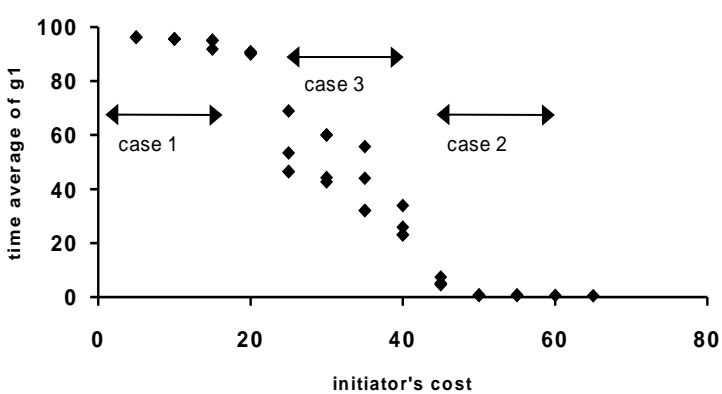

(d)

Figure 1: (a) Typical evolution of cooperation in a population of 800 individuals structured in groups of 50 individuals when cost coefficients are small, showing $g_{l m}, g_{2 m}$ and their standard deviations across the population; (b) same evolution in a single group of 800 individuals ; (c) same evolution as in (a), with intermediary value for $C_{l}$; (d) time average values of $g_{I m}$ in experiments like (a) for several values of $C_{l}$ 

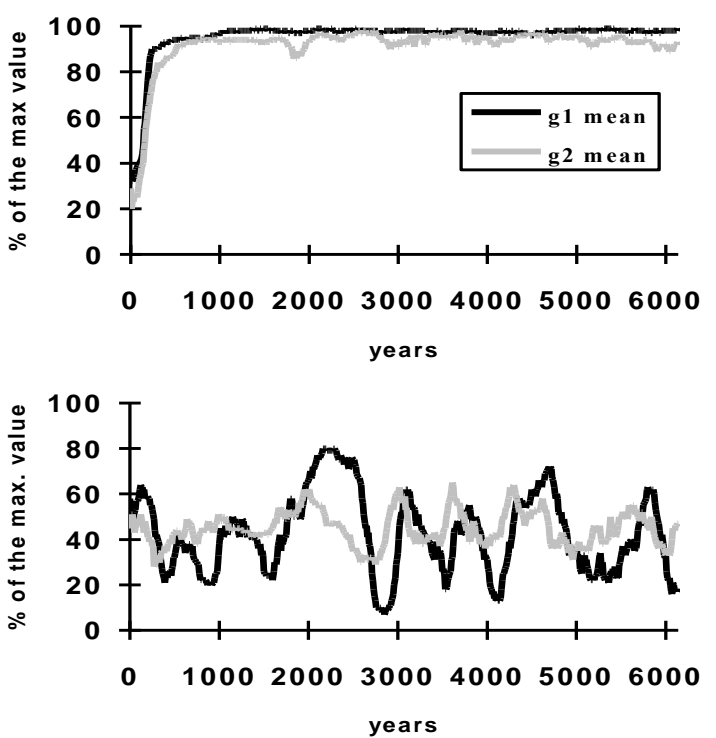

(a)

(b)

Figure 2: Evolution of cooperation in groups of 10 individuals for a $5 \%$ (a) and a $30 \%$ (b) migration rate $\left(C_{I}\right.$ is given a significant value to avoid the convergence observed figure 1a).

random variations? Ferriere and Michod (1996) study dynamic aspects of cooperation, but they focus on transitory phenomena like the spatial expansion of cooperative clusters. The present model allows us to study the evolution of different levels of cooperation over time. It reveals that theoretical equilibria may prove to be of little practical interest because the population's behavior widely fluctuates around them.

The preceding subsection shows that cooperation may be evolutionary stable if some conditions are met. In Case 1 , cooperation stabilizes at a level $g_{2}{ }^{*}$ which is sufficient to encourage high investments $g_{l}$. Temptation to cheat is small. If we put expression (2.5) back into (2.4), we obtain the following payoff:

$$
N P g_{1 m} G \frac{s(1)}{1+\frac{s^{\prime}(1)}{s(1)}}
$$

This is what cooperation provides to optimally responding individuals. This expression should be compared with what uncooperative individuals (carrying $g_{2}=0$ ) would gain: $N P g_{1 m} G s(0)$. The presence of $s^{\prime}(1) / s(1)$ in the above expression, which decreases the global payoff, is the price paid for having a higher probability, measured by $s(1)$, to be chosen. We understand why the level of cooperation at equilibrium is controlled by $s^{\prime}(1)$ (see expression (2.5)): respondents must sacrifice a portion of their potential gains to increase their probability to be chosen as partner.

Case 1, however, presupposes very favorable conditions with large gift and reward coefficients $G$ and $R$ and low cost coefficients $C_{1}$ and $C_{2}$ (see expression 
(2.6)). Moreover, initiators' discernment $s^{\prime}(1)$ must be significant. This latter hypothesis is sometimes taken as granted in some models of the evolution of cooperation (e.g., Cooper and Wallace 1998). We should bear in mind, however, that $s^{\prime}(1)$ is limited by the random aspects of the partner selection procedure. A key issue is to know whether the situation of Case 1 (highly profitable, low cost cooperation with good partner selection) can represent cases of biological altruism. It presupposes that animals need each other to obtain substantial gains that they are not able to reach alone. Maybe cooperative hunting among chimpanzees (Boesch 1996) can be explained this way. Hunting provides energetic food to all members of the hunting team, and cheating (e.g., taking more than one's share of meat) gives only a small immediate relative profit.

This leads us to a further comment about Case 1. Let us consider the global benefit of the population, as a sum of all individual average profits (we suppose the population in equilibrium):

$$
P N g_{1 m}\left[\left(R g_{2 m}-C_{1}\right)+\left(G-C_{2} g_{2 m}\right) s(1) P\right]=P N g_{1 m}\left[\left(R-C_{2}\right) g_{2 m}+\left(G-C_{1}\right)\right]
$$

(at equilibrium, $s(1)=1 / P$ ). If we replace $g_{1 m}$ and $g_{2 m}$ by their values at equilibrium, we get:

$$
\begin{aligned}
P N g_{1 \max }[(R & \left.\left.-C_{2}\right) g_{2}{ }^{*}+\left(G-C_{1}\right)\right] \\
& =P N g_{1 \max }\left[\frac{\left(R-C_{2}\right) G}{C_{2}\left(1+s(1) / s^{\prime}(1)\right)}+\left(G-C_{1}\right)\right] \\
& =P N g_{1 \max }\left[\left(\frac{G R}{C_{1} C_{2}\left(1+s(1) / s^{\prime}(1)\right)}-1\right) C_{1}+\frac{G}{\left(1+s^{\prime}(1) / s(1)\right)}\right]
\end{aligned}
$$

The condition (2.6) insures that both terms of the expression in brackets are positive. This means that individuals are able to generate resources through cooperation that they would not obtain through individual activities. In other words, Case 1 is characterized by the existence of some highly profitable trade between individuals. Such a trade is typically expected between specialized parties who can exchange the product of their activity. We do not expect symmetrical trade between equivalent agents. Moreover, since we are dealing with reciprocal cooperation as an explanation of non-kin altruism, such trade is exposed to cheating, requiring that individuals have enough cognitive abilities to discriminate reliable partners. All these requirements severely limit the range of applicability of Case 1.

If we abandon these restrictions and consider situations in which $G R / C_{1} C_{2}$ is not especially high or that partner selection is not efficient, we enter Case 3. By definition, this situation is expected to have a much broader range of applications. We observed, however, that it leads to important fluctuations in the cooperation level. It is important to grasp the origin of these fluctuations and their consequences on genetic evolution.

At the beginning of each 'year', or after an unsatisfactory encounter, initiators engage in random encounters. These risky trials allow them to discover valuable partners. At the same time, it opens the door to free-riders (individuals with low $g_{2}$ ) who take advantage of being chosen by chance without bearing all the 
costs of cooperation. The intrusion of non-cooperative individuals increases risks for initiators. We thus expect both respondents and initiators to diminish their bids. However, when good cooperators are rare $\left(g_{2 m}\right.$ low $)$, the free-rider strategy is no longer the better strategy. The number of good cooperators increases, offering an opportunity for good initiators to become numerous again. This is why we observe oscillations like those shown in Figure 1c. The reason why the free-rider strategy success depends on the value of $g_{2 m}$ is essentially due to the fact that cooperation is only profitable if the hope of being selected more often as partner outweighs the cost of cooperation. This 'hope' is measured by $s\left(g_{2} / g^{\prime}{ }_{2 m}\right)$. If $g^{\prime}{ }_{2 m}$ is too large, this is not possible. The cross-dependence between $g_{2}$ and its average value $g_{2 m}$ creates an oscillatory regime.

We can draw one important conclusion from the analysis of Case 3. In this unstable situation, cooperation leads to no selection pressure. The standard deviations shown in Figure 1c remain significant, showing that several strategies are simultaneously possible. This result is known from other studies (Frean 1996). Both the instability of strategies and the coexistence of several of them reveal that there is no selection pressure in such a system. Any evolutionary improvement of a given strategy, consisting in a different investment or in a better selection of partners, is soon obsolete and is forgotten in subsequent generations. In other words, neither complex behavior nor biological disposition devoted to cooperation can be expected to emerge.

The fact that simple strategies do not stabilize does not preclude that more complex strategies could emerge and evolve. However, the theoretical search for such complex stable strategies is still unsuccessful. Moreover, even if such strategies existed, one would still have to explain how they could suddenly emerge. If they do not result from an elaboration of simpler and stable cooperative strategies, their hypothetical emergence is very improbable indeed.

\section{Altruism in the absence of cooperation}

The preceding development shows that cooperative altruism can only occur under very specific circumstances, in which some highly profitable trade is possible between two individuals. Moreover, the individual who initiates the trade must be able to check the partner's reliability. These restrictive conditions lead Zahavi to suggest that many observed cases of biological altruism between nonrelated individuals cannot be the result of reciprocal cooperation.

\subsection{ZAHAVI'S THEORY OF PRESTIGE}

Field observations by Zahavi are indeed at odds with the theory of cooperation. The most striking example is perhaps when animals are seen literally to compete to be altruistic, while trying to prevent other individuals being altruistic themselves. In some social bird species like babblers, top ranking males make every effort to feed other members of the group; they displace each other to stand as sentinel on top of a tree; when mobbing a raptor, they risk coming closer to it than their mobbing partners (Zahavi and Zahavi 1997). All these acts are disconnected from any possibility of direct reciprocation:

"If guarding were based on reciprocity, there would be no point in striving to do more guard duty than others. Even if one asserts that such competition 
is necessary to ensure that the group is never without a sentinel, one would still have to explain why each bird interrupts the watch of the one nearest to it in rank, rather than attempting to replace younger, more inexperienced babblers." (Zahavi and Zahavi 1997:135).

We thus need another mechanism to explain how these strange altruistic behaviors could be selected in the phylogenetic past of these species. Zahavi makes the suggestion that in these cases, animals gain prestige and status by behaving altruistically.

"A babbler who can stand guard longer than its comrades, give them part of its food, approach a raptor, take the risk of sleeping at the exposed end of the row - and can also prevent others from doing such deeds - proves daily to its comrades its superiority over them. By doing so, that individual increases its prestige and has an easier time exerting control" (Zahavi and Zahavi 1997:144)

This, indeed, explains why altruistic behavior takes the form of a competition. In a variety of species, high status is a direct or indirect requirement for reproduction, by offering better opportunities to mate, to get more food, to obtain a safer rest place, etc.

Zahavi connects this theory of prestige to the broader framework of his handicap principle. This principle predicts that if altruistic acts are a means of getting prestige, they must be costly in order to be honest. Babblers do not indeed hesitate to engage in costly activities: they take real risks when serving as sentinel or lose opportunities to feed when sharing food. Being costly, their behavior is hard to fake: an overcautious sentinel is not a sentinel, and food sharing cannot be simulated. This aspect of Zahavi's theory does not concern us here. It addresses a form of cheating performed by individuals pretending to be altruistic. We limit ourselves to the study of honest altruistic behavior and investigate whether the theory of prestige can be a sound explanation of it.

Zahavi's handicap principle may explain the strange behavior of babblers if we introduce the concept of quality: only high quality birds can afford to spend energy in wasteful altruistic acts. Altruism would be a way to advertise one's quality in order to attract mates. Such accounts have been proven sound in the frame of sexual selection (Grafen 1990). We will not consider this explanation, even if it could be relevant to the specific case of babblers, for three reasons. First, Grafen takes for granted the fact that females should prefer high quality males. However, since high quality is balanced by a heavy handicap, for the father as for potential male children, the female strategy is not so obviously profitable. Second, the handicap principle does not predict why the birds should behave altruistically. Any wasteful and conspicuous behavior would be convenient. The third reason for discarding the handicap principle here is that sexual selection predicts strong dimorphism. Whenever we observe a characteristic shared by males and females, sexual selection cannot be considered a valid explanation. Several cases of non-kin altruism, especially among humans, are indeed not gender specific. The reality of babblers' ethology, for our purpose here, is not determinant. The example is rather meant as a way to illustrate how a theory of prestige may function. However, Zahavi does not mention any qualitative difference between genders in the babblers' altruistic behavior. We will thus 
follow Zahavi's proposal to consider the theory of prestige as a tentative explanation of the babblers' non-kin altruistic acts.

At first sight, the theory of prestige seems self-consistent. Status is indeed highly profitable for individuals able to acquire it:

"Greater prestige ensures an individual a bigger share of the partnership's 'gains' - that is, a better chance to reproduce successfully." (Zahavi and Zahavi 1997:149)

If status is so advantageous and if it is obtained by performing altruistic acts, then we expect animals to compete for being most helpful in a way which is consistent with Zahavi's observations. Unfortunately, the questions raised by this scenario are as problematic as what is claimed to be solved. How status is related to altruistic acts remains obscure. Zahavi does not give much explanation.

"Prestige reflects the degree of a superior individual's dominance, as recognized by subordinate members of the group. In other words, prestige is gauged by others. The dominant may claim prestige, but for the prestige to be real it has to be accepted by subordinates, and it is this acceptance that actually determines an individual's prestige." (Zahavi and Zahavi 1997:144)

What we must bear in mind is that the fact of granting status to other members of the group is not directly profitable. On the contrary. How can individuals get any genetic interest in awarding prestige to conspicuously altruistic individuals? The latter should be indeed positively reinforced, since they behave for the good of other individuals or of the whole group, but this cannot be a justification for individual acts of gratitude. Members who accept to bestow better access to food or to mates on a given individual do it at their own expense. As Zahavi puts it:

"Increased prestige for one partner means a loss in prestige for another. In other words, it is a zero-sum game within the group." (Zahavi and Zahavi 1997:149)

Gratefulness behavior is open to deception: deceivers would wait for altruistic individuals to compete to win the altruistic battle, let others grant them status, and themselves deny any status to the winner. By adopting this ungrateful strategy, an individual increases its own reproduction expectancy: it will not only avoid giving the lion's share to other individuals, it will also give less of a chance to them to get a higher status than its own. Under such circumstances, grateful attitudes which allow some individuals to gain precedence seem inexplicable.

The scenario proposed by Zahavi, if narrowly interpreted, does not significantly differ from the cooperation scenario that he rightfully criticizes. Individuals are altruistic in the hope of being rewarded with status. However, if altruistic acts are costly, renouncing part of one's own reproductive expectancy to reward altruistic fellows is costly too. Even if it does not look like cooperation, the theory of prestige is not unlike it, since it relies on a trade as well. The scenario is open to deception. We thus do not expect any stable behavior to emerge, either from the altruistic side or from the thankful respondents. We will first illustrate this negative result by simulating a weak version of Zahavi's proposal. In Section 4, we show that thanks to additional 
hypotheses, Zahavi's scenario can be extended to form a sound explanation of some forms of non-kin altruism.

\subsection{IMPLEMENTING ZAHAVI'S THEORY OF PRESTIGE}

A simplified model of the theory of prestige can be based on the following hypotheses:

- (H1) Individuals: Individuals are characterized by two gradual genes. The value $g_{l}$ of the first one determines the propensity to display some behavior $\mathrm{H}$. The value $g_{2}$ of the second gene determines the propensity to give status to other individuals. Each individual has an intrinsic ability $q$ to perform $\mathrm{H}$, randomly determined at birth. This ability has no genetic basis. An individual's performance on $\mathrm{H}$ is thus $q g_{l} n$, where $n$ is a noise modulation. This performance induces a cost $C_{1} q g_{1} n$ where $C_{l}$ is a constant.

- (H2) Interactions: Individuals interact on several occasions each 'year'. During an interaction, an individual A performs $\mathrm{H}$ while being observed by $\mathrm{B}$. B will grant status to A on the basis of A's performance on $\mathrm{H}$. H is optionally altruistic, i.e., B may benefit from A's performance by the amount $G q_{A} g_{1 A} n$, where $G$ is a constant.

- (H3) Status: Status is randomly initialized each 'year'. When B observes A's performance, A's status is increased by the amount $R g_{2 B} q_{A} g_{1 A} n$, where $R$ is a constant. There may be a corresponding cost supported by B: $C_{2} g_{2 B} q_{A} g_{1 A} n$, where $C_{2}$ is a constant.

- (H4) Success: At the end of each 'year', status is converted into life points for each individual in the population ( $S$ life points for one status point).

The status of a given individual at the beginning of a 'year' is represented by a random variable $\theta$. At the end of the 'year', we sum over all interactions initiated by this individual to obtain its expected status:

$$
\theta+\sum_{i} \operatorname{Rg}_{2 i} q g_{1} n_{i}=\theta+N R g_{2 m} q g_{1}+\varphi_{1}
$$

where $g_{2 m}$ is the local average of $g_{2}$ and $N$ the number of interactions initiated per individual per year. $g_{2}$ and the noise $n$ are not correlated. If $N$ is large, $\sum_{i} g_{2 i} n_{i}$ is approximately $N g_{2 m}$ (the average of $n$ is 1 ). The random variable $\varphi_{1}$ is introduced here to represent the error due to the fact that $N$ is limited. It is centered (zero mean). The expected profit of a given individual, considering only the initiator's strategy, results from the cumulated amount of status earned during interactions, and from the cost associated with the production of $\mathrm{H}$ :

$$
S\left(\theta+N R g_{2 m} q g_{1}+\varphi_{1}\right)-\Sigma_{i} C_{1} q g_{1} n_{i} \cong N q g_{1}\left(S R g_{2 m}-C_{1}\right)+S\left(\theta+\varphi_{1}\right)
$$

Individuals may directly benefit from others' performance during interactions:

$$
\sum_{i} G q_{i} g_{l i} n_{i}=N G q_{m} g_{1 m}+\varphi_{2}
$$




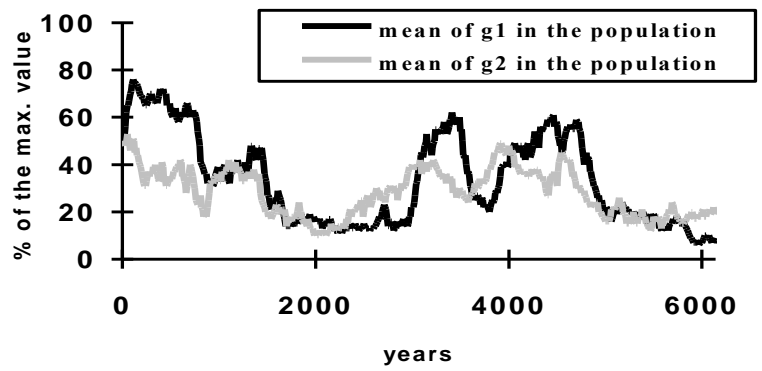

Figure 3: A simple version of Zahavi's theory of prestige does not show convergence. In the experiment plotted here, costs $C_{1}$ and $C_{2}$ are given small values.

where $\varphi_{2}$ is a centered random variable and $g_{l m}$ is the local average of $g_{1}$. The introduction of the respondent's strategy adds the corresponding cost:

$$
-\Sigma_{i} C_{2} g_{2} q_{i} g_{1 i} n_{i}=-N C_{2} g_{2} q_{m} g_{l m}+\varphi_{3}
$$

where $q_{m}$ and $g_{l m}$ are the average values of $q$ and $g_{l}$, and $\varphi_{3}$ a random variable. The global profit is thus, on average:

$$
\left.N\left[q g_{1}\left(S R g_{2 m}-C_{1}\right)+\left(G-C_{2} g_{2}\right) q_{m} g_{l m}\right)\right]+\Phi
$$

where $\Phi$ represents the random part of the profit $\left(S\left(\theta+\varphi_{1}\right)+\varphi_{2}+\varphi_{3}\right)$. If we ignore $q$ (by making $q=1$ ), formula (3.1) is an instance of the cooperation model : it looks exactly like the sum of expressions (2.1) and (2.2). There is a difference, though. In the present model, there is no memory of cooperative respondents. $s^{\prime}(1)$ is thus zero in (2.5). As a consequence, there is no tendency to grant status is this scenario. Figure 3 illustrates the phenomenon. High values of $g_{l}$ are of course profitable as long as there are individuals who accept to grant status. But as soon as $g_{l m}$ increases, $g_{2 m}$ tends to decrease. Figure 3, though it was computed for low values of $C_{I}$ and $C_{2}$, shows no convergence.

Zahavi's account of altruistic behavior, if limited to 'prestige' alone, is not sufficient to explain why altruism is evolutionary stable. We will see, however, that thanks to additional hypotheses, this scenario can constitute a sound explanation of altruism. We will define a 'political' situation in which altruism and status allowance coevolve.

\section{Altruism, status and politics}

The purpose of this section is not to claim that there is one single sound explanation of altruism among non-related individuals. It is rather to show that there exists at least one such explanation, based on Zahavi's notion of prestige, which does not rely on cooperation. 


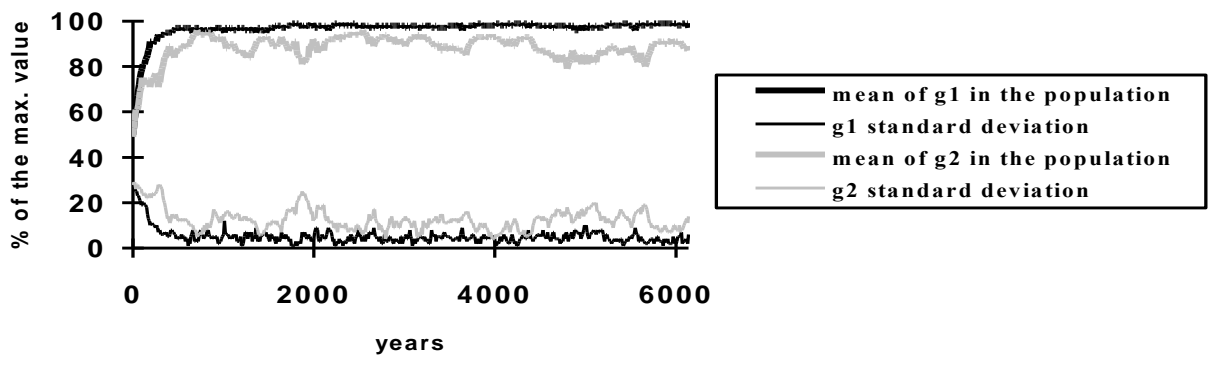

Figure 4: $\quad$ Convergence of the 'political' model. This typical plot is obtained when the value of $S R$ is sufficient (see figure 6).

\subsection{A 'POLITICAL' MODEL OF ALTRUISM}

The problem is to find a context in which altruism and status allowance coevolve by reinforcing each other. Situations of coalition formation are such a context. We make the following additional hypotheses:

- (H5) Coalitions: Within a group, individuals may join to form coalitions. B joins A's coalition if A's performance on $H$ is better than all what $B$ experienced in the current 'year'. These coalitions are dissolved each 'year' and must be constituted anew. Interactions take place preferentially within the coalition, but with some probability, with any member of the group.

- (H6) Politics: Each 'year', coalitions undergo a competition. Coalitions are evaluated according to the ability $q^{*}$ of their top-ranking individual, i.e., the individual with highest status. Their relative aptitude $r$ is $r=q^{*} / q_{M}$, where $q_{M}$ is the average coalition aptitude.

- (H7) Success: Individuals' status is affected by the relative aptitude of their coalition, i.e., it is multiplied by $\sigma(r)$ where $\sigma$ is a linear or sigmoid function between 0 and $1 . \sigma(r)$ is called coalition factor.

The coalition factor $\sigma(r)$ affects the computation of status. Formula (3.1), which gives the profit of a given individual, becomes:

$$
\left.N\left[q g_{1}\left(\operatorname{SR} \sigma(r) g_{2 m}-C_{1}\right)+\left(G-C_{2} g_{2}\right) q_{m} g_{1 m}\right)\right]+\Phi(r)
$$

The random part $\Phi$ now depends on the coalition aptitude. The situation now departs from the cooperative scenario. Respondents keep a memory of good initiators by entering their coalition. It was the reverse in the cooperative scenario. But the fundamental difference between both models lies in the presence of the coalition factor $\sigma(r)$. As we will see, it is this factor that allows for convergence.

\subsection{CONVERGENCE OF THE 'POLITICAL' MODEL}

If $S$ and $R$ are sufficiently large while $C_{1}$ and $C_{2}$ remain limited, we observe that both $g_{1}$ and $g_{2}$ reach high values (Figure 4). This model offers us a situation in which altruism and status allowance coevolve.

We may wonder why $g_{2}$ tends to increase, since it only appears in a negative 
term in (4.1). In fact, $\sigma(r)$ also depends on $g_{2}$. The top-ranking individual is determined as the individual with maximum status:

$$
\max _{i}\left[\theta+v_{i} q+\varphi\right] ; \quad v_{i}=N \operatorname{Ng}_{2 m} g_{1 i}
$$

$v_{i}$ is the vote intensity in favor of individual $i$ (rigorously, the mean is computed without considering individual $i$ ). We see that if $g_{2 m}$ is low, the random part of the term to be maximized may become prevalent. As a consequence, the top-ranking individual is likely to be a member with an average ability. Such a bad choice for $q$ will lead to a poor value for $\sigma(r)$, since the coalition is likely to lose the competition (hypothesis H6). Expression (3.1) then becomes largely negative. Thus if $g_{2 m}$ is low, there is a high probability of losing points for everybody in the coalition. It is in the interest of each member to locally increase $g_{2 m}$ by increasing its own $g_{2}$. If we focus on a given member B of the coalition carrying a gene $g_{2}$, expression (4.2) can be rewritten as:

$$
\max _{i}\left[\theta+v_{i} q+\varphi\right] ; \quad v_{i}=N R g_{2 m} g_{1 i} ; \quad g_{2 m}=(1-\alpha) g_{2 m}^{\prime}+\alpha g_{2}
$$

$g^{\prime}{ }_{2 m}$ is the average of $g_{2}$ computed when excluding B and $\alpha$ is the proportion of the interactions that individuals in the coalition have with B. Notice that during the 'year', individuals may interact with individuals outside their coalition and may change coalition when they observe superior performance on $\mathrm{H}$. Interactions most often take place within the coalition, but for one part, the status obtained by an individual results from interactions outside the coalition. The coalition relevant in (4.3) is the coalition to which B belongs by the end of the 'year'. We see that $g_{2}$ plays the role of a voting weight. We will call $g_{2}$ the vote factor. With large values for $g_{2}$, individuals make use of their knowledge of each other's performance $\left(q g_{1 i}\right)$ to decide who will represent the coalition in the competition. In other words, a large $g_{2}$ increases the probability that $\sigma(r)$ will be high. This explains why $g_{2}$ converges to a significant value (Figure 4).

Formula (4.1) shows that as soon as $\sigma(r)$ is high enough to allow the term $\left(S \sigma(r) R g_{2 m}-C_{l}\right)$ to be positive, individuals with large $g_{l}$ do better by increasing their expected profit. In other words, the genetic strategy which consists, with a large $g_{l}$, in displaying its true own ability $q$ is profitable as soon as there are individuals who are ready to reward it in terms of status. We thus understand why $g_{l}$ reaches maximal value, as shown in Figure 4.

As expected, this scenario predicts that both $g_{1}$ and $g_{2}$ will evolve toward significant values. There are however a few points that must be made clear to see how the model radically differs from a cooperative model. The preceding explanations are straightforward for coalitions which have some hope, by having made the right choices, of obtaining a good $\sigma(r)$. However, $\sigma(r)$ gives an advantage only to better than average coalitions. This means that about half of the coalitions will get a low $\sigma(r)$. It seems that when there is a probable loss, individuals with low values for $g_{l}$ and $g_{2}$ would do better, since they would at least cancel the costs. Moreover, it seems that status allowance is altruistic. Why not let other members in the coalition lose energy (cost term with $C_{2}$ ) and relative success in awarding status around them (increasing the status of others indeed automatically diminishes one's own relative success)? After all, if others make correct choices, it is preferable to spare voting costs by having a small $g_{2}$. 
These arguments, which predict that $g_{1}$ and $g_{2}$ will not rise, are valid when $C_{l}$ or $C_{2}$ are too large, compared with the coefficient $S R$ which affects $\sigma(r)$ in formula 5'. However, when $S R$ reaches a significant value, having a large $g_{l}$ is a good way of making substantial gains. To understand, this, we must consider the average benefit of $g_{1}$ and $g_{2}$ over generations. Coalitions change every 'year' and individuals are replaced as generations depart. A gene like $g_{l}$ will be carried by many individuals over time. On average over generations, such a lineage will get the following benefit (the number of generations considered is supposed large enough for the coupling between $g_{1}$ and $g_{2}$ to be negligible and for $g_{2}$ to average on $g_{2 m}$ ):

$$
\begin{aligned}
& N\left[q_{m} g_{1}\left(S R \sigma_{m} g_{2 m}-C_{1}\right)+\left(G-C_{2} g_{2 m}\right) q_{m} g^{\prime}{ }_{1 m}\right]+\Phi_{m} \\
& \quad=N\left[g_{1}\left(S R g_{2 m} / 2-C_{1}\right)+\left(G-C_{2} g_{2 m}\right) g^{\prime}{ }_{1 m}\right] / 2+\Phi_{m}
\end{aligned}
$$

$q$ and $\sigma(r)$ are averaged to $1 / 2$, since they do not depend on $g_{l} ; \Phi_{m}$ is the mean of the random variable $\Phi(r) ; g^{\prime}{ }_{l m}$ stands for the mean of $g_{l}$ over other members, excluding the considered lineage. We see that the decision for $g_{l}$ crucially depends on $g_{2 m}$. It is, however, an all-or-nothing decision. If $g_{2 m}$ reaches a certain threshold that makes $\left(S R g_{2 m} / 2-C_{l}\right)$ positive, $g_{l}$ will tend to maximal values.

We must consider now what could lead $g_{2 m}$ to increase and reach this threshold. If we consider the benefit (4.1) over generations for a given lineage carrying the gene $g_{2}$, we get:

$$
\begin{aligned}
& N\left[q_{m} g_{1 m}\left(S R \sigma_{m} g_{2 m}^{\prime}-C_{1}\right)+\left(G-C_{2} g_{2}\right) q_{m} g_{1 m}\right]+\Phi_{m} \\
& \quad=N\left[\left(S R \sigma_{m} g_{2 m}^{\prime}-C_{1}\right)+\left(G-C_{2} g_{2}\right)\right] g_{l m} / 2+\Phi_{m}
\end{aligned}
$$

We distinguish $g^{\prime}{ }_{2 m}$ from $g_{2 m}$ since the average status obtained by a lineage results from $g_{2}$ averaged on other individuals, excluding the lineage itself. $g_{2 m}^{\prime}$ does not depend on $g_{2}$. However, the average value of $\sigma(r), \sigma_{m}$, does. The relative aptitude $r$ of a coalition indeed depends on the local average vote factor to which $g_{2}$ contributes (see (4.3)). Expression (4.5) thus shows that there is a competition between two terms, $S R \sigma_{m} g^{\prime}{ }_{2 m}$ and $-C_{2} g_{2}$. The gist of the model lies in the fact that the benefit $S R \sigma_{m} g^{\prime}{ }_{2 m}$ depends non-linearly on $g_{2}$, while the cost $-C_{2} g_{2}$ is linear.

The value of $\sigma_{m}$ depends on the correct choice expressed in (4.3). Figure 5a shows the probability of making a correct choice within a coalition, depending on the average vote intensity $v_{m}=N R g_{2 m} g_{1 m}$. When $v_{m}$ is zero, then the probability of selecting the individual with the highest $q$ is $1 / M$, where $M$ is the average size of coalitions. When $v_{m}$ increases, the probability that the individual with highest $q$ is selected tends toward 1 . This is due to the fact that in the latter case, the random part in expression (4.2) is dominated by the 'vote' $v_{i} q$. Figure 5 b shows, according to $v_{m}$, typical coalition factors $\sigma_{m}$ associated with the probabilities of Figure $5 \mathrm{a}$. These values are computed in a context where the perfect strategy (best individual always selected) is prevalent in the remainder of the population (hence the fact that $\left.\sigma_{m}<0.5\right)$. Figure $5 \mathrm{c}$ shows the expression in square brackets in (4.5) as a function of $g_{2}$ :

$$
f\left(g_{2}\right)=\left[\left(S R \sigma_{m} g_{2 m}^{\prime}-C_{1}\right)+\left(G-C_{2} g_{2}\right)\right]
$$




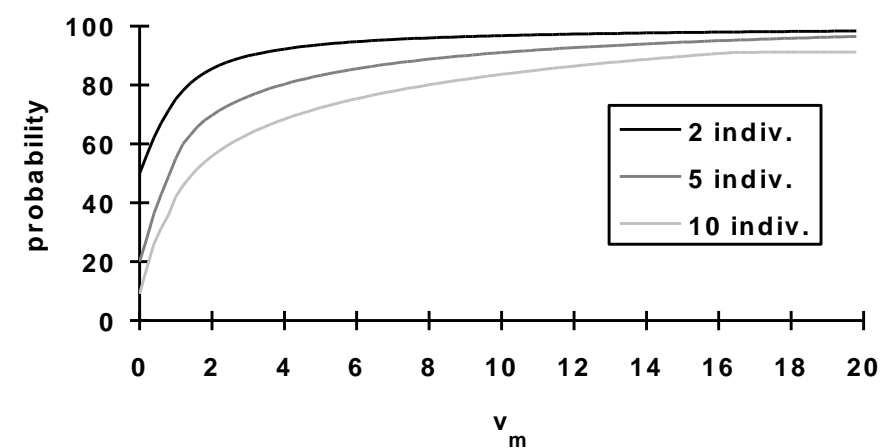

(a)

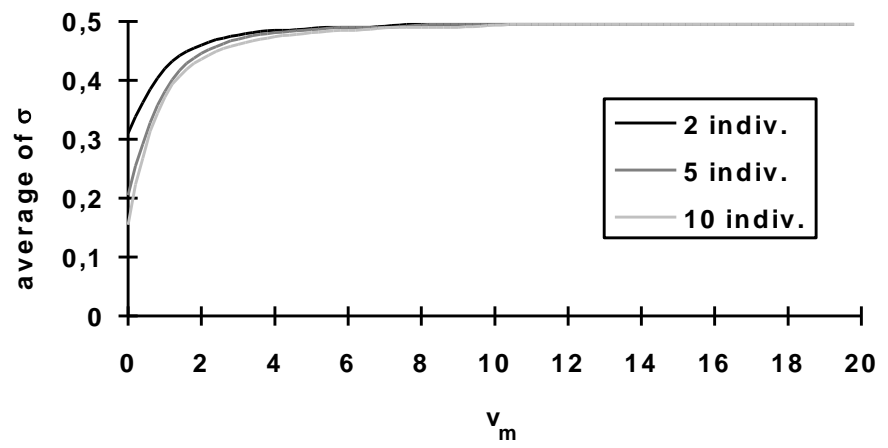

(b)

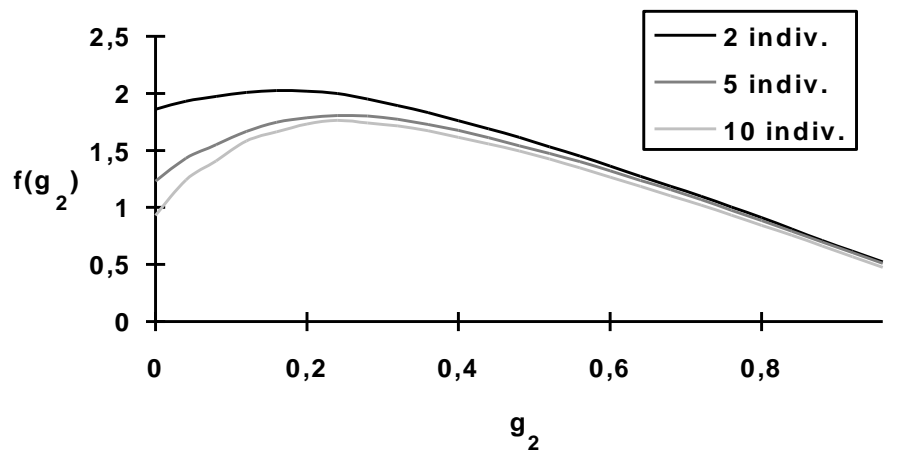

(c)

Figure 5: Study of the coalition factor. (a) shows the probability of choosing the best individual in the population according to the vote intensity $v_{m}$. (b) shows the corresponding coalition factor $\sigma_{m}$. In (c), the function $f$ is plotted against $g_{2}$. The probability $\alpha$ is 0.2 , which leads to an expansion of the $\mathrm{x}$-axis of a factor 5 . 
( $\sigma_{m}$ depends on $g_{2}$ through $v_{m}=N R g_{1 m}\left((1-\alpha) g_{2 m}^{\prime}+\alpha g_{2}\right)$, as in (4.3)). As we will see in the next subsection, the vote factor $g_{2}$ will evolve toward the maximum of this curve and stabilize there.

It is important to note that the existence of a stable maximum is due to the nonlinearity of the coalition factor $\sigma_{m}$, which is itself the result of the intrinsic nonlinearity of the voting system. The probability of selecting a good candidate to represent the coalition in the competition increases in a non-linear way when $g_{2}$ augments. For two-member coalitions, this probability is easily computed (we assume that $\theta$ and $q$ have a uniform density on $[0,1])$ :

$$
\left(6+4 v_{m}-v_{m}{ }^{2}\right) / 12 \text { when } 0<v_{m}<1 ; 1-1 /\left(3 v_{m}\right)+1 /\left(12 v_{m}{ }^{2}\right) \quad \text { when } v_{m}>1
$$

The slope for small values of $v_{m}$ is 4 (see Figure $5 \mathrm{a}$ ). The derivatives of the relative coalition aptitude $r$ and the coalition factor $\sigma(r)$ are expected to be even higher, as suggested by Figure $5 \mathrm{~b}$. When these derivatives exceed $C_{2} / \mathrm{NRg}_{1 \mathrm{~m}} \alpha$, we are certain to have a stable maximum for an intermediary value of $g_{2}$, as shown Figure 5c.

\subsection{Stability of the ALtruism For Status trade}

We presented a model which is able to predict a stable altruistic strategy, balanced by a stable status allowance strategy. It is important to compare the conditions of stability of this model with those of the cooperative model. We will show that there is one evolutionary stable strategy, represented by the pair $\left(g_{\text {lmax }}\right.$, $\left.g_{2}{ }^{*}\right)$, where $g_{l \max }$ is the maximal value of $g_{1}$. This strategy is close to $\left(g_{1 \max }, g_{2}{ }^{0}\right)$, where $g_{2}{ }^{0}$ is the abscise of the maximum of $\left.f(g 2)\right)$. By definition, $f^{\prime}\left(g_{2}{ }^{0}\right)=0$. The maximum of expression (4.5) is obtained for a value $g_{2}{ }^{*}$ such that:

$$
f^{\prime}\left(g_{2}^{*}\right)=\frac{-f\left(g_{2}^{*}\right)}{g_{1 m}} \cdot \frac{\partial g_{1 m}}{\partial g_{2}}
$$

(we neglect the dependence of $\Phi_{m}$ on $g_{2}$ ). If we suppose that $\partial g_{1 m} / \partial g_{2}$ is small and $g_{1 m}$ large, $f^{\prime}\left(g_{2}{ }^{*}\right)$ must be small. We have locally $f^{\prime}\left(g_{2}{ }^{*}\right)=\left(g_{2}{ }^{*}-g_{2}{ }^{0}\right) f^{\prime \prime}\left(g_{2}{ }^{0}\right)$. The factor $f^{\prime \prime}\left(g_{2}{ }^{0}\right)$ has a significant (negative) value, due to the non-linearity of $\sigma_{m}$ (cf. Figure $5 \mathrm{c})$. As a consequence, $\left|g_{2}{ }^{*}-g_{2}{ }^{0}\right|$ must be small. Under such circumstances, the maximum of (4.5) is thus obtained for a value close to $g_{2}{ }^{0}$.

Now consider expression (4.4). As long as the term $\left(\mathrm{SRg}_{2 \mathrm{~m}} / 2-\mathrm{C}_{1}\right)$ remains positive, $g_{l}$ will not drift away from $g_{l \max }$. We are thus in a situation of double bind which guarantees stability. Let us start from $g_{I m}=g_{1 \max }$ and $g_{2 m}=g_{2}{ }^{0}$. If the domain where $\left(\mathrm{SRg}_{2}{ }^{\mathrm{O}} / 2-\mathrm{C}_{1}\right)>0$ is not too narrow, we expect $g_{2}$ to have very little effect on $g_{l m}$, since there will be no selection pressure to push $g_{l}$ away from $g_{\text {Imax }}$. As we saw, $g_{I m}$ large and $\partial g_{I m} / \partial g_{2}$ small ensure that $g_{2}{ }^{*}$ remains close to $g_{2}{ }^{0}$. This, in turn, satisfies the constraint $\left(\operatorname{SRg}_{2 m} / 2-C_{l}\right)>0$, since at equilibrium $g_{2 m}=g_{2}{ }^{*}$. So neither $g_{1 m}$ nor $g_{2 m}$ will drift away from $g_{1 m a x}$ and $g_{2}{ }^{0}$.

The condition for the existence of an equilibrium is thus:

$$
\left(\operatorname{SRg}_{2}{ }^{0} / 2-C_{1}\right)>0
$$

Expression (4.6) shows that $g_{2}{ }^{0}$ must have a significant value, and that the 


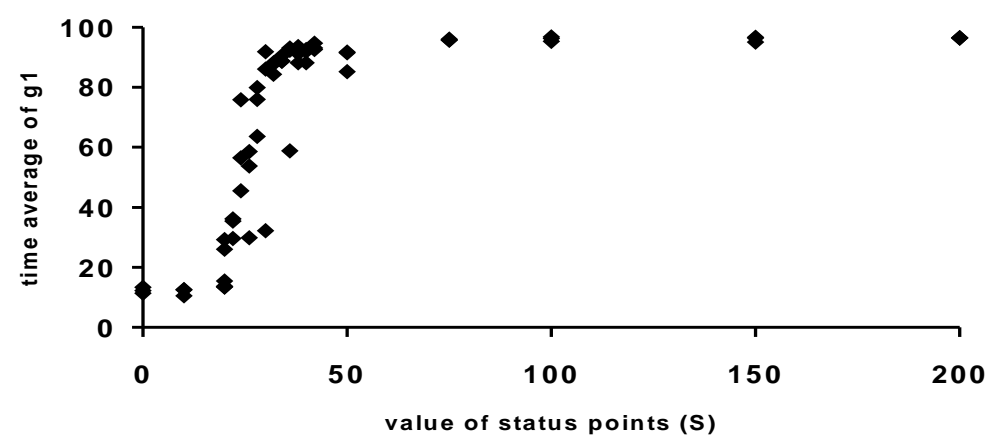

Figure 6: $\quad$ Influence of the status-to-vitality conversion coefficient $S$. Analysis performed through 64 runs of 3000 generations each. For each run, the time average of $g_{l}$ is computed and its value is plotted. Experiments have been concentrated around the cut-off value.

status reward and its conversion into 'life points' must be large enough to compensate for the cost associated with the display of $\mathrm{H}$. The cost coefficient $C_{2}$ is implicit in (4.6), since $g_{2}{ }^{0}$ depends on it. This expression predicts that the 'political' scenario will converge when coefficients $R$ and $S$, which control the production of status and its conversion into vitality, are high enough while costs $C_{1}$ and $C_{2}$ remain limited. Figure 6 confirms this prediction: reliable convergence does exist above a cut-off value for the coefficient $S$.

Expression (4.6) should be compared with expression (2.6) which gives the conditions of stability in the cooperative scenario. There are however three important differences between both scenarios. The first difference comes from the $\operatorname{cost} C_{2}$, which controls the stability of altruism in the two scenarios. However, the meaning of this coefficient is not exactly the same in both cases. $C_{2}$ determines in one case the price paid for being cooperative, and in the other case the direct cost for granting status $\left(C_{2}\right.$ does not include the indirect handicap generated by the fact of advantaging others). Both acts are not equivalent, though. Cooperation is material, it requires a tangible expense of matter or energy to be effective. By contrast, status allowance can be immaterial, there is no reason for it systematicallyto involve a direct significant cost. The constraint of a low $C_{2}$ is thus much easier to satisfy in the 'political' scenario.

The second difference comes from cheating detection, which was an essential component of the cooperative scenario. Individuals making the first step must have some confidence in the fact that their partners will reciprocate. In the 'political' model, the situation is inverted. If there is cheating at all, it is now expected from the first individual's side, while cheating detection should be performed by the second individual. It is indeed essential to the model that status be granted on the basis of a performance which correlates with a genuine political ability. If some individuals are able to simulate $\mathrm{H}$, they gain status even if they lack the required abilities to really perform $\mathrm{H}$, and the whole system collapses. As a consequence, $\mathrm{H}$ must be hard to fake, either by being costly (Zahavi 1995) or because other individuals have sufficient discriminative abilities to avoid deception. 
A consequence of this inverted situation is that $\mathrm{H}$ can be performed toward several individuals simultaneously, which was impossible in the face-to-face cooperative scenario. We may think that there is still a possibility of cheating by failing to grant status to good performers, especially when other individuals can do the job. This is not, however, a profitable strategy. The coalition factor $\sigma(r)$ introduces an indirect component into the trade. There is no point to cheating: a lower $g_{2}$, by locally making $g_{2 m}$ a bit too small, has bad consequences for all members of the coalition by significantly affecting $\sigma(r)$, and the cheating individual suffers from the situation it created.

The third important difference to be mentioned between the present model and the cooperative model is related to the global economy of the system. Let us consider the sum of all profits (4.1) made during a 'year' in a group of size $P$ :

$$
\left.P N\left[g_{1 m}\left(S R g_{2 m} / 2-C_{1}\right) / 2+\left(G-C_{2} g_{2 m}\right) g_{1 m} / 2\right)\right]
$$

At equilibrium, we have $g_{2 m} \approx g_{2}{ }^{0}$ :

$$
P N g_{1 m} / 2 \cdot\left[\left(\operatorname{SRg}_{2}{ }^{0} / 2-C_{1}\right)+\left(G-C_{2} g_{2}{ }^{0}\right)\right]
$$

From (4.6), we must conclude that the first term within the brackets is positive. The presence of the second term, $\left(G-C_{2} g_{2}{ }^{0}\right)$, makes the situation quite different from what we had in the cooperation model. The most striking difference is that the global balance needs not be positive. The constant $G$ does not play any role in the computation of the equilibrium $\left(g_{\operatorname{lmax}}, g_{2}{ }^{0}\right)$. $G$ may even be negative. This means that, depending on ecological settings, $H$ may be altruistic, but that it need not always be so. When $G$ is zero, $\mathrm{H}$ may take the form of competitive displays, like the babbler "dance" (Zahavi and Zahavi 1997). When $G$ is small or negative, or when $C_{2}$ is significant, the overall profit may be small or negative. This means that the 'political' model does not require the production of a significant amount of additional resources, contrary to the cooperative model. The model is however fully compatible with a situation of altruistic display, with significant values for $G$. The 'political' model is not limited by the symmetry hypothesis, which is a basic assumption of the cooperation model. In some cases, we may expect that individuals will devote energy to provide a profusion of wealth in the hope of getting status. Notice that direct altruism $(G>0)$, though compatible with the model, is not predicted by it. However, the model requires that the ability to perform $\mathrm{H}$ be correlated with coalition success. The ability to improve the security or welfare of a group may show such a correlation. This may be the reason why babblers are so altruistic. Although it is an ethological issue, we may conjecture that these birds, to some extent, choose to live in the group of individuals who show their ability to perform efficient altruistic acts. Zahavi describes the inter-group competition among babblers as a struggle, and the life of solitary individuals in open desert as hazardous. In such a context, the ability to join a successful group is essential. Losing welfare and reproduction opportunities in exchange for security may prove advantageous if it is done by granting status to efficient groupmates, i.e., individuals showing the ability to significantly increase, through their sole behavior, the probability of success of the group. Ethological descriptions made by Zahavi about babblers let us suppose that the rewarded 
abilities: guarding, feeding of low ranking individuals or predator mobbing, when performed by one or a few individuals, may have a definite influence on the fate of the whole coalition. This is what our 'political' model requires.

These three differences between the cooperative model and the 'political' model give them different ranges of applicability. Whenever costs are non-symmetrical, when cheating detection is performed by respondents, or when there is no evidence of any profitable trade generating additional resources, reciprocal cooperation cannot be invoked and the 'political' model becomes a good candidate of non-kin altruism.

\subsection{Discussion: IMPLICATIONS AND LIMITATIONS OF THE MODEL}

The scenario described here relies on the presence of a new level, a competition between coalitions, that we name 'politics'. This scenario is very different from the introduction of an authority, like a government, which discourages individuals from cheating during private interactions by means of dissuading penalties (Axelrod 1984:156). The existence of an authority may stabilize cooperation, as long as cheating detection and dissuasion are efficient. We do not postulate such a supra-individual mechanism. However, it is possible to combine our scenario with this kind of policed cooperation scenario: the behavior $\mathrm{H}$ could consist in performing policing acts. This is an altruistic behavior which, in some settings, could correlate with coalition success. In such cases, policing would be rewarded with status and cooperative acts could be performed with confidence. This suggests that cooperation may evolve and stabilize in a 'political' context. Apart from this specific situation, there is no a priori link between our hypothesized 'political' level and any enforcement of honest cooperation.

Our scenario also differs from situations in which coalition members merely undergo a collective risk or may hope for a collective benefit. Most kinds of collective effects do not suppress the possibility of cheating. When computing the derivative of genetic cost/benefit balance, we observe that the derivative of the collective benefit must compensate for the negative derivative of the cost. As discussed above, the existence of a stable situation requires that the marginal collective effect is significantly positive for small individual investments. This is definitely the case in our coalition scenario where the voting process provides immediate benefits, as Figure $5 \mathrm{~b}$ illustrates. This would not be the case, however, for a simple collective effect in which groups compete, for instance, by comparing the cumulative wealth of their members. In this case, the marginal influence of individual cooperation on the collective success is poor and would not compensate for the marginal cost.

Our 'political' scenario is not a mere instance of 'group selection', for two reasons. First, coalitions are not groups, since members of a coalition at least in part chose each other on the basis of their performance on H. Second, the individual marginal influence on coalition effect is significant. This is not the case for usual collective effects. For these two reasons, cheating, which is always profitable in 'group selection', is not a valid strategy in our scenario.

The original assumption of the 'political' model is that the same characteristic $q$ that gives an advantage in the competition between coalitions allows to obtain status from conspecifics (hypothesis H5). The individual advantage of granting 
status to others according to their performance lies in the higher probability of being well represented in the competition. Individuals with a large $g_{2}$ reward high $q$ individuals by proportionally granting them status. As a consequence, those individuals are likely to represent the coalition. Since their $q$ is high, the coalition is likely to obtain a high $\sigma(r)$. Hence $\sigma_{m}$ will be higher than average over generations. This link between $g_{2}$ and $\sigma_{m}$ is an essential part of the model. If we suppress this correlation, then $\partial \sigma_{m} / \partial g_{2}$ is zero instead of being significantly positive, and thus $g_{2}$ goes down to zero.

The model presented here has been designed to show how an evolutionary stable strategy of altruistic behavior can emerge. Several of its aspects are of course oversimplified:

- Direct costs and benefits are supposed to be linear. It would be interesting to study the validity of the model when this is not the case.

- The competence $q$ of individuals in performing $\mathrm{H}$ has no genetic basis. This restriction was introduced to focus on the evolution of the genetic 'willingness' $\left(g_{l}\right)$ to produce $\mathrm{H}$. We did not experiment with a genetically based competence. Notice, however, that as long as $g_{l}$ and $q$ are not correlated, our results should not be affected, even if $q$ is inherited.

- Individuals do not differ in their willingness to join good performers. In other words, the principle of coalition formation is postulated in the model. This willingness to follow other individuals could have a genetic basis. Beyond the emergence of actual coalitions, which does occur in the model, we could thus obtain an emerging sociality.

- Individuals have very limited cognitive abilities. They only remember the best performance previously observed. Of course, much cognitive performance is implicitly assumed in the fact that individuals are supposed to behave in competing coalitions. As in models of cooperation in which complex individual strategies are sometimes studied, individual behaviors could depend on more complex structures than the mere factors $g_{1}, g_{2}$ and $q$.

- Competition between coalitions is merely assumed, it is neither modeled nor given an opportunity to emerge. In a more complete simulation, the coalition effect could be an emerging consequence of specific socio-ecological settings.

- The notion of coalition effect is excessively simple. A coalition's success depends on the sole best ranking individual's competence. A more realistic account could then involve a more complex computation of the coalition effect. The model would thus be extended to account not only for simplified 'politics', but for simplified 'friendship' as well.

Despite all these limitations, the present model relies on a robust principle that allows non-cooperative altruism to emerge. The core of the model, which is that the 'voting' process provides a non-linear benefit to individuals, remains valid through all the above suggested improvements. Even if we learn much from them, these developments are likely to confirm that a non-linear collective effect is able to stabilize rewarded altruism. The 'voting' process resulting from status allowance may be extended to other non-linear processes. We may thus come upon a new theory of sociality among non-related individuals. 


\section{Conclusion: biosociality revisited}

The first purpose of this paper was to dismiss cooperation as the ultimate explanation of altruism among non-related individuals. Cooperation may exist under very specific conditions, when some one-to-one trade is profitable to both partners and when individuals making the first step are able to detect uncooperative partners. When profits are poor or when cheating detection is not efficient enough, the free-rider strategy, which is not advantageous when there are few cooperative individuals, becomes prevalent as soon as the population reaches a certain level of cooperation. This leads to a complex dynamics in which random fluctuations are sustaining an oscillatory regime. This situation cannot generate any selection pressure.

When all the conditions for the existence of cooperation are not met, non-kin altruistic behavior remains a mystery. This is the case for instance when the altruistic behavior is directed toward several individuals simultaneously, or when no possibility of reciprocation is offered. The second objective of this paper was to propose a consistent model that may explain such forms of altruism. At first sight, the 'political' model presented here may seem quite peculiar: it is based on the emergence of coalitions and on a system of help-for-status trade. This model, which we designated as 'political', indeed leads to an evolutionary stable equilibrium. On the one hand, individuals compete in displaying a certain behavior that can be altruistic. On the other hand, they reward good performers by granting them status. This apparently reciprocal altruism is made stable by the existence of a competition between coalitions. Individuals who group around good performers and designate them as such are likely to win this inter-coalition contest.

In real politics, those who want to be in the victorious camp should join the side of the most promising candidate and designate her/him as their champion. During political campaigns, candidates behave rather altruistically, they spend a lot of money, and their supporters accept to give them the leadership, whatever the consequences after the election. This is also the essence of the mechanism invoked in our model. Of course, as an operational model of biological altruism, this is grossly simplified and many less caricatured scenarios can be proposed.

When Wilson described Human Nature, he could only conceive of two evolutionary explanations of sociality: kin selection, which is commonly invoked to explain eusociality in insect societies, and reciprocal altruism, which is claimed to operate mostly in primate and human societies (Wilson 1978:156). We showed that the latter mechanism is questionable. The model presented in Section 4 shows that there is at least one further alternative. It extends Zahavi's observation of the role of prestige and status as motives for altruistic performance. Zahavi's explanation, however, lacks an account of the willingness to grant status. This disposition, in itself, is as altruistic as the rewarded behavior. Its evolutionary stability is thus not predicted. In our 'political' model, however, status allowance appears as a profitable strategy: it plays the role of a vote. The stake of being well represented in the competition may justify the cost of conferring status upon others.

Our 'political' model was of course intended as an attempt to design a sound explanation of some forms of altruism, not as a restrictive account of all forms of altruistic interactions among non-related individuals. It may however give us a new insight into several bio-sociological phenomena, including some aspects of 
primate or human social behavior. Temporary coalitions to take over have been described among chimpanzees (Goodall 1971; de Waal 1982). This ability to form political coalitions is also an obvious human characteristic. In the context of our model, this behavior is evolutionarily sound as soon as the criterion for joining a coalition is correlated with its political success.

Let us consider a behavior taken as example by Wilson: heroism. In all cultures, heroes, i.e., individuals who prove their ability to perform courageous, though sometimes futile, acts, are conspicuously honored. Sociobiology (Wilson 1975, 1978) can only account for one part of the story: trying to appear as a hero is a profitable strategy since it provides a better access to resources and reproduction. Sociobiology cannot explain at all why other individuals, by honoring heroes, grant them with such essential advantages. Within the frame of the model described in Section 4, we may see this instance of human social behavior as an extreme case of coalition effect. Heroic acts are not necessarily directly profitable to observers $\left(G\right.$ low), though they are very risky $\left(C_{1}>>1\right)$. Why do heroes deserve status? According to the model, the ability to be a hero must be correlated with the success of the coalition. In other words, it should be advantageous to be friend of courageous individuals, because it helps to have them in the coalition during the inter-coalition contest. If we accept this statement, then the model provides an evolutionary account for heroism, which is not the case of sociobiology.

In (Dessalles 1998; in press), we suggest that a similar account may apply to the evolution of language, though this time $G$ is positive and $C_{l}$ is small. The basic idea is that when coalitions reach a certain size, as hypothesized for human ancestors, relevant information replaces physical strength as determinant of success in the inter-coalition contest.

Sociality among unrelated individuals, which is best exemplified by the human species, deserves a sound explanation that reciprocal altruism is unable to provide. We designed a new model in which altruism can be a stable strategy. Beyond mere altruism, it predicts the existence of many complex social behaviors organized around a specific status system. In many animal species, status is extorted by brute force. It seems that among humans, and possibly in species like babblers, status is willingly granted. This opens the door to a new range of social behaviors that our species seems to have widely explored.

\section{References}

Axelrod, R., and Hamilton, W. D. (1981). "The evolution of cooperation". Science, 211, 1390-1396.

Axelrod, R. (1984). The Evolution of Cooperation. New York: Basics Books.

Boesch, C. (1994). "Hunting strategies of Gombe and TaÏ Chimpanzees". In R. W. Wrangham, W. C. McGrew and F. B.M. de Waal (eds), Chimpanzee cultures. Harvard University Press, 77-91.

Cooper, B. and Wallace, C. (1998). "Evolution, partnerships and cooperation". Journal of Theoretical Biology, 195, 315-328.

Dawkins, R. (1976). Le gène égoïste. Editions Menges, ed. 1978.

de Waal, F.B.M. (1982). Chimpanzee politics: power and sex among apes. Baltimore : The John Hopkins Univ. Press, ed. 1989.

Dessalles, J-L. (1996). L'ordinateur génétique. Paris : Hermès. 
Dessalles, J-L. (1998). "Altruism, status, and the origin of relevance". In J. R. Hurford, M. Studdert-Kennedy and C. Knight (eds), Approaches to the Evolution of Language - Social and Cognitive Bases. Cambridge University Press, 130-147.

Dessalles, J-L. (to appear). "Language and hominid politics". In C. Knight, J. R. Hurford and M. Studdert-Kennedy (eds), The evolutionary emergence of language: social function and the origin of linguistic form (to appear). Cambridge : Cambridge University Press.

Dugatkin, L. A. (1997). "The evolution of cooperation - Four paths to the evolution and maintenance of cooperative behavior". Bioscience, 47(4.2), 355-362.

Dunbar, R. I. M. (1996). Grooming, gossip, and the evolution of language. Cambridge : Harvard University Press.

Ferriere, R. and Michod, R. E. (1996). "The evolution of cooperation in spatially heterogeneous populations". The american naturalist, 147(3.1).

Frean, M. (1996). "The Evolution of Degrees of Cooperation". Journal of Theoretical Biology, 182(2.6).

Goldberg, D. E. (1989). Genetic algorithms in search, optimization and machine learning. Addison Wesley Publishing Company.

Goodall, J. (1971). In the Shadow of man. Boston : Houghton Mifflin Company, ed. 1988.

Grafen, A. (1990). "Biological signals as handicaps". Journal of Theoretical Biology, 144, 517-546.

Hamilton, W. D. (1964). "The genetical evolution of social behavior". The Journal of Theoretical Biology, 7, 1-16.

Jones, D. (1996). "Varieties of group selection". Behavioral and Brain Sciences, 19(2.6), 778 .

Key, C. and Aiello, L. (to appear). A prisoner's dilemma model of the evolution of paternal care.

Macy, M. W. and Skvoretz, J. (1998). "The evolution of trust and cooperation between strangers: a computational model". American Sociological review, 63, 638-660.

Nakamaru, M., Matsuda, H. and Iwasa, Y. (1997). "The Evolution of Cooperation in a Lattice-Structured Population". Journal of Theoretical Biology, 184(1).

Palmer, C. T., Fredrickson, B. E. and Tilley, Cristopher (1996). "Group selection or categorical perception?". Behavioral and Brain Sciences, 19(2.6), 780.

Peck, J. R. (1993). "Friendship and the Evolution of Cooperation". Journal of Theoretical Biology, 162(2), 195-228.

Trivers, R. L. (1971). "The evolution of reciprocal altruism". The Quaterly Review of Biology, 46.

Williams, G. C. (1966). Adaptation and natural selection: a critique of some current evolutionary thought. Princeton : Princeton University Press.

Wilson, D. S. and Sober, E. (1994). "Reintroducing group selection to the human behavioral sciences". Behavioral and Brain Sciences, 17, 585-654.

Wilson, E. O. (1975). Sociobiology: the new synthesis. Harvard University Press.

Wilson, E. O. (1978). On human nature. Cambridge, MA : Havard University Press. 
Zahavi, A. (1995). "Altruism as a handicap - the limitations of kin selection and reciprocity". Journal of Avian Biology, 26(1), 1-3.

Zahavi, A. and Zahavi, A. (1997). The handicap principle. New York : Oxford University Press. 